\title{
A!
}

This is an electronic reprint of the original article.

This reprint may differ from the original in pagination and typographic detail.

Laakso, Mikko; Dowhuszko, Alexis A.; Wichman, Risto

\section{Empirical evaluation of OFDM waveforms for VLC in the presence of LED nonlinearities}

Published in:

2021 IEEE 32nd Annual International Symposium on Personal, Indoor and Mobile Radio Communications, PIMRC 2021

DOI:

10.1109/PIMRC50174.2021.9569542

Published: 13/09/2021

Document Version

Peer reviewed version

Please cite the original version:

Laakso, M., Dowhuszko, A. A., \& Wichman, R. (2021). Empirical evaluation of OFDM waveforms for VLC in the presence of LED nonlinearities. In 2021 IEEE 32nd Annual International Symposium on Personal, Indoor and Mobile Radio Communications, PIMRC 2021 (pp. 465-470). (IEEE International Symposium on Personal, Indoor and Mobile Radio Communications). IEEE. https://doi.org/10.1109/PIMRC50174.2021.9569542

This material is protected by copyright and other intellectual property rights, and duplication or sale of all or part of any of the repository collections is not permitted, except that material may be duplicated by you for your research use or educational purposes in electronic or print form. You must obtain permission for any other use. Electronic or print copies may not be offered, whether for sale or otherwise to anyone who is not an authorised user. 


\title{
Empirical evaluation of OFDM waveforms for VLC in the presence of LED nonlinearities
}

\author{
Mikko Laakso, Alexis A. Dowhuszko, Senior Member, IEEE, and Risto Wichman \\ School of Electrical Engineering, Aalto University, 02150 Espoo, Finland \\ Email: \{mikko.t.laakso, alexis.dowhuszko, risto.wichman\}@aalto.fi
}

\begin{abstract}
Visible Light Communications (VLC) rely on LED light sources to transmit information wirelessly, offering an alternative air interface to contemporary mobile networks using Radio Frequency bands for wireless connectivity. Most commercial LEDs found in the market nowadays have been designed for illumination, showing a notable non-linear response when performing the Electrical-to-Optical (E/O) conversion, particularly when using deep Intensity Modulation (IM) indexes for increased useful radiated power. Several different OFDM-based waveforms, adapted to the unipolarity requirement that IM demands, have been proposed in the literature. However, the performance of most of these Optical OFDM waveforms is seriously affected by the non-linear magnitude distortion of the low-cost white LEDs, particularly when the Peak-to-Average Power Ratio (PAPR) is high. To tackle this issue, this paper analyzes the performance of Constant-Envelope (CE) OFDM, a waveform that modulates the phase of an Intermediate Frequency (IF) carrier with a real-valued OFDM signal before its $\mathrm{E} / \mathrm{O}$ conversion. The Bit Error Rate of CE-OFDM is compared to the one of DC-biased Optical OFDM and passband OFDM using a software-defined VLC demonstrator. CE-OFDM allows the VLC system to work in energy-efficient regimes, thanks to its robustness to the nonlinear magnitude distortion introduced by the LED.
\end{abstract}

Index Terms-VLC, Nonlinear distortion, DCO-OFDM, CEOFDM, Phosphor-Converted LEDs, Software-defined demo.

\section{INTRODUCTION}

Visible Light Communication (VLC) has strong potential to complement the wireless connectivity services that mobile networks have provided so far exclusively on Radio Frequency (RF) bands [1]. VLC tackles many challenges that RF-based communications currently face, particularly in ultradense indoor deployments. For example, VLC can easily mitigate the inter-cell interference using highly-directive spotlights, improve the security of the communication by confining the light signal into the desired coverage area, and save energy by reusing the same optical power for both communications and illumination services. In addition, VLC uses a portion of the electromagnetic spectrum that is by definition licence-free, and relies on low-cost mass-produced and energy-efficient LEDs as transmitters, which reduce the implementation costs of VLC systems notably [2]. Unfortunately, LEDs have a strong non-linear forward voltage versus output radiant flux transfer relation, which may impact notably the VLC link-level performance if this impairment is not properly addressed [3].

As the LEDs need a real-valued and positive (i.e., unipolar) input voltage to perform the Electrical-to-Optical (E/O) conversion, baseband complex-valued OFDM signals must be adapted to the transmission over the VLC link. Several waveforms have been presented in the literature to fulfill this VLC requirement [4]. One of the most popular ones is DCbiased Optical (DCO)-OFDM, in which the input vector to the Inverse Fast Fourier Transform (IFFT) is Hermitian symmetric, and a DC level is added to the real-valued output signal before clipping the negative values [5]. However, this is not the approach initially followed in standardization. For example, in the amendment IEEE 802.11bb for light communications [6], the upconversion of the complex baseband OFDM signal to a suitable Intermediate Frequency (IF) is being considered to reuse the available Wi-Fi chipsets. However, both waveforms are sensitive to the nonlinear distortion caused by the LEDs.

The biasing point of the LED transmitter should be carefully tuned to maximize the forward current, while minimizing the clipping probability to mitigate the amplitude distortion of the VLC transmission [7]. However, due to the large Peak-to-Average Power Ratio (PAPR) that OFDM signals experience [5], [8], optimal DC-bias and AC-scaling factors that keep under control the LED nonlinear distortion - while optimizing the fraction of the total radiant flux that is emitted for communications - are difficult to identify. The effect of nonlinear-distortion can be mitigated by pre-distortion [7], post-distortion [9], or a combination of both techniques [3], which increase the complexity of the VLC transceivers. Instead, we study Constant Envelope (CE)-OFDM, a waveform with low PAPR [10], [11]. The effect of the LED non-linear distortion for the three OFDM waveforms under study is modeled theoretically following a similar analysis as in [12], and their performance is empirically assessed using a softwaredefined VLC demonstrator. Based on the obtained results, we show that CE-OFDM outperforms DCO-OFDM and passband OFDM, particularly when the LED is biased to work beyond its linear region for energy efficiency purposes.

The rest of the paper is organized as follows: Section II introduces the system model and derives the signal model for the three different OFDM waveforms under consideration, namely DCO-OFDM, passband OFDM, and CE-OFDM. Section III characterizes the impact of the LED non-linear distortion and derives the closed form expressions that model this impairment. Bit Error Rate (BER) measurements for the three different OFDM-based waveforms are presented in Section IV. Finally, conclusions are drawn in Section V.

\section{SYSTEM MODEL}

The block diagrams for the three OFDM-based VLC transmissions under consideration are illustrated in Fig. 1. Here, 

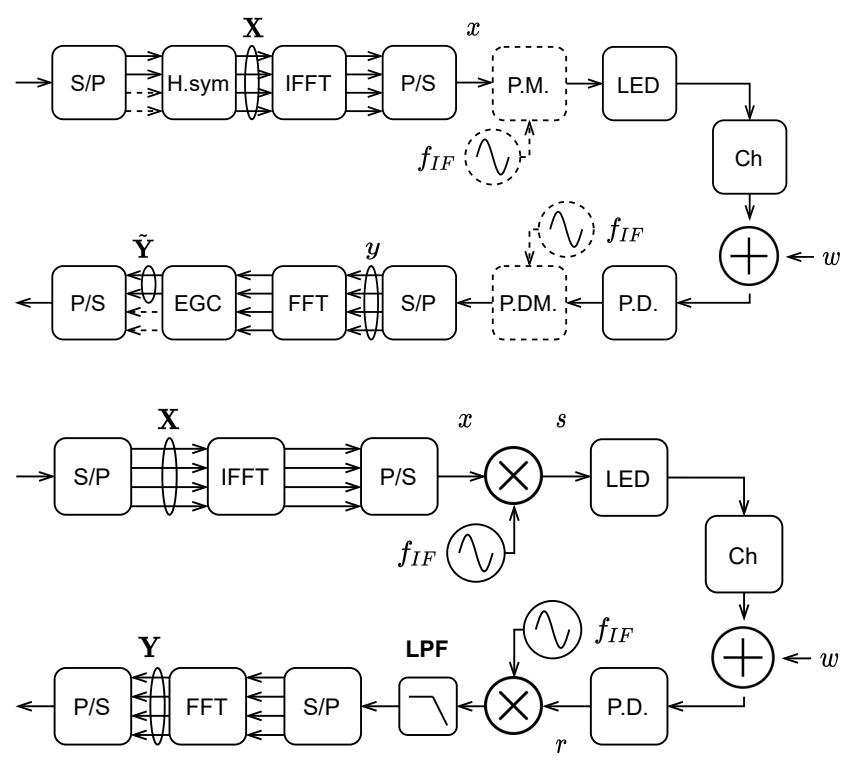

Fig. 1: Simplified transmitter and receiver block diagrams for DCOOFDM and CE-OFDM (upper subfigure) and passband OFDM (lower subfigure). For CE-OFDM, the Phase Modulation (PM) and Phase Demodulation (PDM) blocks are marked with dashed lines.

the input QAM symbols are adapted to modulate the light intensity of the LED, before the optical wireless channel $(\mathrm{CH})$ atenuates the signal and adds noise $(w)$. At the receiver side, signal processing is applied to the signal samples at the output of the Photodetector (PD), before QAM symbols are detected. In the following subsections, the signal model for each of these three OFDM-based waveforms are described in detail.

\section{A. Signal model for DCO-OFDM transmission}

The input bit stream is divided into groups of $m$ bits and, after that, they are Gray mapped onto $M=2^{m}$ points of a $M$-QAM constellation. The Hermitian Symmetric feature is then introduced on the $N$-size vector that feeds the IFFT, i.e.,

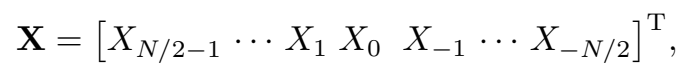

verifying

$$
X_{k}= \begin{cases}X_{-k}^{*} & k=1, \ldots, N / 2-1 \\ 0 & k=0,-N / 2 .\end{cases}
$$

This way, the time domain signal at the IFFT output, i.e.,

$$
x[n]=\frac{1}{\sqrt{N}} \sum_{k=-N / 2}^{N / 2-1} X_{k} \exp \left(\mathrm{j} \frac{2 \pi k n}{N}\right), \quad n=0, \ldots, N-1,
$$

becomes real-valued as the imaginary part is always zero.

The non-linear response of the phosphor-converted white LED is modeled with a third-order polynomial function, i.e.,

$$
g_{\mathrm{LED}}(x)=c_{3} x^{3}+c_{2} x^{2}+c_{1} x+c_{0},
$$

where the coefficients $c_{i}$ depend on the DC polarization point and AC-scaling gain that is selected for the LED driver circuit. Then, the received time-domain at the PD output becomes

$$
y[n]=g_{\mathrm{LED}}(x[n]) / L+w[n],
$$

where $L$ is the path loss attenuation between the LED transmitter and PD receiver, and $w[n]$ is the Additive White Gaussian Noise (AWGN) term that includes the thermal noise introduced by the Transimpedance Amplifier (TIA) and the effect that the background illumination have on the received signal.

The Fast Fourier Transform (FFT) is then applied to the sequence of received signal samples, obtaining

$$
Y_{k}=\frac{1}{\sqrt{N}} \sum_{n=0}^{N-1} y[n] \exp \left(-\mathrm{j} \frac{2 \pi k n}{N}\right), \quad k=-N / 2, \ldots, N / 2-1 .
$$

Finally, the Equal Gain Combining (EGC) processing

$$
\tilde{Y}_{k}=\left(Y_{k}+Y_{-k}^{*}\right) / \sqrt{2}, \quad k=1, \ldots, N / 2-1,
$$

is utilized to exploit the Hermitian symmetric structure of the vector of transmit symbols in (1), before $M$-QAM detection is used to estimated the transmitted symbols $\widehat{X}_{k}$.

\section{B. Signal model for passband OFDM transmission}

For passband OFDM, the Hermitian Symmetry requirement (2) on the symbol vector $\mathbf{X}$ is removed; therefore, each OFDM symbol accommodates $(N-1)$ QAM symbols. Then, after the IFFT processing of (3) and the upconversion into the IF, the passband OFDM signal becomes

$$
\begin{aligned}
s(t) & =\operatorname{Re}\left\{\sum_{n=0}^{N-1} x[n] h_{\mathrm{tx}}\left(t-n T_{\mathrm{s}}\right) \exp \left(\mathrm{j} \omega_{\mathrm{IF}} t\right)\right\} \\
& =a(t) \cos \left(\omega_{\mathrm{IF}} t+\phi(t)\right), \quad \omega_{\mathrm{IF}}=2 \pi f_{\mathrm{IF}},
\end{aligned}
$$

where $T_{\mathrm{S}}$ is the symbol time, $f_{\mathrm{IF}}$ is the intermediate frequency, $h_{\mathrm{tx}}(t)$ is the response of the transmit pulse shaping filter, and

$$
\begin{aligned}
& a(t)=\left|\sum_{n=0}^{N-1} x[n] h_{\mathrm{tx}}\left(t-n T_{\mathrm{s}}\right)\right| \\
& \phi(t)=\arg \left\{\sum_{n=0}^{N-1} x[n] h_{\mathrm{tx}}\left(t-n T_{\mathrm{s}}\right)\right\}
\end{aligned}
$$

are the instantaneous amplitude and phase that the envelope of the IF carrier takes, respectively. Then, after downconversion and sampling at the symbol rate, the received signal becomes

$$
y[n]=g_{\mathrm{LED}}(a[n] \exp (\mathrm{j} \phi[n])) / L+w[n] .
$$

After the FFT processing in (6), the symbol detection block estimates the transmitted $M$-QAM symbols, $\widehat{X}_{k}$. Note that for passband OFDM, there is no EGC block as the symbol vector $\mathrm{X}$ does not verify the Hermitian symmetric requirement.

\section{Signal model for CE-OFDM transmission}

In CE-OFDM, the real-valued time-domain signal that modulates the phase of the IF subcarrier is obtained by verifying the Hermitian Symmetric feature (2) in the symbol vector $\mathbf{X}$, before feeding this sequence into the IFFT processing (3). After that, the phase modulated signal becomes

$$
s(t)=A_{0} \cos \left(\omega_{\mathrm{IF}} t+\phi_{\mathrm{pm}}(t)\right)
$$


where $A_{0}$ is the constant amplitude of the IF carrier and

$$
\phi_{\mathrm{pm}}(t)=k_{\mathrm{pm}} \sum_{n=0}^{N-1} x[n] h_{\mathrm{tx}}\left(t-n T_{\mathrm{s}}\right)
$$

is the phase shift that that the real-valued modulating signal introduces in the IF carrier when using the phase modulation index $k_{\mathrm{pm}}[\mathrm{rad} / \mathrm{V}]$. Then, the received signal attains the form

$$
r(t)=\frac{g_{\mathrm{LED}}\left(A_{0}\right)}{L} \cos \left(\omega_{\mathrm{IF}} t+\phi_{\mathrm{pm}}(t)\right)+w(t)
$$

and, after phase demodulation, the received samples become

$$
\begin{aligned}
& y[n]=\left(\phi_{\mathrm{pm}}[n]+\varepsilon[n]\right) / k_{\mathrm{pm}}, \quad w[n]=|w[n]| \exp \left(\mathrm{j} \phi_{w}[n]\right),(15) \\
& \varepsilon[n]=\tan ^{-1}\left\{\frac{|w[n]| \sin \left(\phi_{w}[n]-\phi_{\mathrm{pm}}[n]\right)}{g_{\mathrm{LED}}\left(A_{0}\right) / L+|w[n]| \cos \left(\phi_{w}[n]-\phi_{\mathrm{pm}}[n]\right)}\right\} \\
& \approx \frac{|w[n]| \sin \left(\phi_{w}[n]-\phi_{\mathrm{pm}}[n]\right)}{g_{\mathrm{LED}}\left(A_{0}\right) / L} \text { for } \frac{g_{\mathrm{LED}}\left(A_{0}\right)}{L} \gg|w[n]| .
\end{aligned}
$$

Finally, after the FFT processing in (6) and EGC in (7), the transmit $M$-QAM symbols are estimated in reception.

\section{THEORETICAL MODELLING OF THE NON-LINEAR DISTORTION INTRODUCED BY THE LED}

The transfer function of the phosphor-converted white LED is modeled with a polynomial function of order three, according to (4). The coefficients of the transfer function $g_{\mathrm{LED}}(\cdot)$ depend on both the DC-level and AC-scaling factor that are selected for the operation of the LED driver. As expected, the larger is the AC-scaling factor, the higher is the portion of the transmitted optical power that is used to transport the data symbols but, in return, the higher is the non-linear distortion power that is introduced. Similarly, the DC-level can adjust the excursion range of the LED radiant flux (or current), such that the upper/lower clipping probabilities are minimized. The non-linear distortion that the LED generates is now studied for the three OFDM-based waveforms presented in Section II.

\section{A. Non-linear distortion modelling in DCO-OFDM}

When the number of subcarriers $N$ grows large, a timedomain OFDM signal can be approximated by a set of independent and identically distributed (i.i.d.) Gaussian Random Variables (RVs) [13]. Then, according to the Bussgang theorem [14], the non-linear distortion that the LED introduces can be described by a gain factor $(\alpha)$ and an additional noise component $(Z)$. In other words, let $X$ be a zero-mean Gaussian $\mathrm{RV}$ and $g(\cdot)$ an arbitrary memoryless distortion on $X$, then

$$
g(X)=\alpha X+Z, \quad \text { with } \mathbb{E}\{X Z\}=0 .
$$

In the previous equation, the gain factor is constant, i.e.,

$$
\alpha=\mathbb{E}\{X g(X)\} / \sigma_{X}^{2}, \quad \sigma_{X}^{2}=\mathbb{E}\left\{X^{2}\right\},
$$

and the additional noise component $Z$ that is added is not correlated with $X$. Then, due to the central limit theorem, the noise $Z$ becomes AWGN in the frequency domain at the FFT output. Therefore, the mean value of the time-domain signal at the output of the LED, i.e. $\mathbb{E}\{g(X)\}=E\{Z\}$, affects only the value of the sample contained on the DC subcarrier, whereas an additional zero-mean AWGN component with variance

$$
\sigma_{Z}^{2}=\mathbb{E}\left\{Z^{2}\right\}-\mathbb{E}^{2}\{Z\}, \quad \mathbb{E}\left\{Z^{2}\right\}=\mathbb{E}\left\{g^{2}(X)\right\}-\alpha^{2} \sigma_{X}^{2}
$$

is added on the signal samples received in the remaining subcarriers. To sum up, the overall effect of the LED nonlinearity $g_{\mathrm{LED}}(\cdot)$ is an increase on the received noise power by $\sigma_{Z}^{2}$, and a decrease of the useful signal power by factor $\alpha^{2}$.

To find $\alpha$ in closed form, we first need to compute the cross correlation between the input and output signals, i.e.,

$$
\begin{aligned}
\mathbb{E}\left\{X g_{\mathrm{LED}}(X)\right\} & =c_{3} \mathbb{E}\left\{X^{4}\right\}+c_{2} \mathbb{E}\left\{X^{3}\right\}+c_{1} \mathbb{E}\left\{X^{2}\right\}+c_{0} \mathbb{E}\{X\} \\
& =3 c_{3} \sigma_{X}^{4}+c_{1} \sigma_{X}^{2},
\end{aligned}
$$

where the coefficients of $g_{\mathrm{LED}}(\cdot)$ are shown in (4). Note that since RV $X$ is approximated as zero-mean Gaussian, the odd raw moments are all zero, whereas the even raw moments attain the form $\mathbb{E}\left\{X^{p}\right\}=\sigma_{X}^{p}(p-1)$ !!, with $p$ !! equal to the double factorial of $p$ (i.e., the product of all numbers from $p$ to 1 that have the same parity as $p$ ). Then, by combining (18) with (20), the following closed form expression is obtained:

$$
\alpha_{\text {dco }}=3 c_{3} \sigma_{X}^{2}+c_{1} \text {. }
$$

The mean value of the signal at the output of the LED, $\mathbb{E}\{g(X)\}$, only affects the DC-subcarrier which, in our system model, is not used to transmit any information. However, when we focus on the rest of the subcarriers, we have that

$$
\begin{aligned}
\mathbb{E}\left\{g_{\mathrm{LED}}^{2}(X)\right\} & =c_{3}^{2} \mathbb{E}\left\{X^{6}\right\}+c_{0}^{2}+2 c_{3} c_{2} \mathbb{E}\left\{X^{5}\right\}+2 c_{1} c_{0} \mathbb{E}\{X\} \\
& +\left(2 c_{3} c_{1}+c_{2}^{2}\right) \mathbb{E}\left\{X^{4}\right\}+\left(2 c_{2} c_{0}+c_{1}^{2}\right) \mathbb{E}\left\{X^{2}\right\} \\
& +2\left(c_{3} c_{0}+c_{2} c_{1}\right) \mathbb{E}\left\{X^{3}\right\} \\
& =15 c_{3}^{2} \sigma_{X}^{6}+\left(6 c_{3} c_{1}+3 c_{2}^{2}\right) \sigma_{X}^{4} \\
& +\left(2 c_{2} c_{0}+c_{1}^{2}\right) \sigma_{X}^{2}+c_{0}^{2} .
\end{aligned}
$$

Then, by combining (19) with (22), the final closed form expression for the additional noise variance becomes

$$
\begin{aligned}
\sigma_{Z}^{2} & =\left[6 c_{3}^{2} \sigma_{X}^{6}+3 c_{2}^{2} \sigma_{X}^{4}+2 c_{2} c_{0} \sigma_{X}^{2}+c_{0}^{2}\right]-c_{2}^{2} \sigma_{X}^{4} \\
& \cong 2 \sigma_{X}^{4}\left(3 c_{3}^{2} \sigma_{X}^{2}+c_{2}^{2}\right) \quad \text { for } c_{0} \cong 0 .
\end{aligned}
$$

\section{B. Non-linear distortion modelling in passband OFDM}

As explained in Section II-B, the received signal here is

$$
\begin{aligned}
r & {[n]=g_{\mathrm{LED}}\left(a[n] \cos \left(\omega_{\mathrm{IF}} t+\phi[n]\right)\right) } \\
& =c_{3} a^{3}[n] \cos ^{3}\left(\omega_{\mathrm{IF}} t+\phi[n]\right)+c_{2} a^{2}[n] \cos ^{2}\left(\omega_{\mathrm{IF}} t+\phi[n]\right) \\
& +c_{1} a[n] \cos \left(\omega_{\mathrm{IF}} t+\phi[n]\right)+c_{0} \\
& =\frac{c_{3} a^{3}[n]}{4} \cos \left(3 \omega_{\mathrm{IF}} t+3 \phi[n]\right)+\frac{c_{2} a^{2}[n]}{2} \cos \left(2 \omega_{\mathrm{IF}} t+2 \phi[n]\right) \\
& +\left(\frac{3 c_{3} a^{3}[n]}{4}+c_{1} a[n]\right) \cos \left(\omega_{\mathrm{IF}} t+\phi[n]\right)+\left(c_{0}+\frac{1}{2}\right) \cdot(24)
\end{aligned}
$$

The authors of [13] provided an extenssion of Bussgang Theorem to bandpass memoryless nonlinearities, which for the case of Gaussian zero-mean stationary inputs attains the form

$$
\alpha=\frac{1}{2} \mathbb{E}\left\{g_{\mathrm{LED}}^{\prime}(a[n])+\frac{g_{\mathrm{LED}}(a[n])}{a[n]}\right\} .
$$


Since a low-pass filter is used in reception after downconversion, we only need to keep the term in (24) that is centered on $\omega_{\mathrm{IF}}$ which, after simple manipulations, is given by

$$
\alpha_{\mathrm{pb}}=\mathbb{E}\left\{(3 / 2) c_{3} a^{2}[n]+c_{1}\right\}=(3 / 2) c_{3} \mathbb{E}\left\{a^{2}[n]\right\}+c_{1},
$$

with

$$
\mathbb{E}\left\{a^{m}[n]\right\}=\int_{0}^{A_{\max }} x^{m} f_{a[n]}(x) d x,
$$

where $A_{\max }$ is the upper clipping amplitude and $m$ is the order or the raw-moment amplitude samples before clipping, whose squared values are exponentially distributed with mean $\sigma_{X}^{2}$. Then, when $A_{\max }=1$, it is possible to show that

$\mathbb{E}\left\{a^{2}[n]\right\}=\sigma_{X}^{2}\left[1-\exp \left(-1 / \sigma_{X}^{2}\right)\right]$,

$\mathbb{E}\left\{a^{4}[n]\right\}=2 \sigma_{X}^{4}\left[1-\exp \left(-1 / \sigma_{X}^{2}\right)\left(1+1 / \sigma_{X}^{2}\right)\right]$,

$\mathbb{E}\left\{a^{6}[n]\right\}=6 \sigma_{X}^{6}\left[1-\exp \left(-1 / \sigma_{X}^{2}\right)\left(1+1 / \sigma_{X}^{2}+1 /\left(2 \sigma_{X}^{4}\right)\right)\right]$.

Similarly, the non-linear distortion noise that is added in the LED can be approximated as [13]

$$
\begin{aligned}
\sigma_{Z}^{2} & =\mathbb{E}\left\{\left|g_{\mathrm{LED}}(X)\right|^{2}\right\}-\alpha_{\mathrm{pb}} \mathbb{E}\left\{X^{2}\right\} \\
& =(9 / 16) c_{3}^{2} \mathbb{E}\left\{a^{6}[n]\right\}+(3 / 2) c_{3} c_{1} \mathbb{E}\left\{a^{4}[n]\right\} \\
& +c_{1}^{2} \mathbb{E}\left\{a^{2}[n]\right\}-\alpha_{\mathrm{pb}}^{2} \mathbb{E}\left\{X^{2}\right\} .
\end{aligned}
$$

\section{Non-linear distortion modelling in CE-OFDM}

The effect of the LED non-linear distortion only affects the amplitude of the phase modulated sub-carrier that is received which, according to the analysis presented in (24), is given by

$$
y[n]=\left((3 / 4) c_{3} A_{0}^{3}+c_{1} A_{0}\right) \cos \left(\omega_{\mathrm{IF}} t+\phi_{\mathrm{pm}}[n]\right)+w[n] .
$$

Therefore, if amplitude $A_{0}=\min \left\{\sqrt{2 \sigma_{X}^{2}}, A_{\max }\right\}$ to make fair comparisons to DCO-OFDM and passband OFDM, the effect of the non-linear LED response in CE-OFDM is a compression of the received signal samples by scaling factor

$$
\alpha_{\mathrm{ce}}=\frac{(3 / 4) c_{3} A_{0}^{3}+c_{1} A_{0}}{c_{1} A_{0}}, \quad \text { where } c_{3}<0 .
$$

Note that there is no additional noise power added here since the LED non-linear distortion takes place in the magnitude of the received signal. Therefore, $\sigma_{Z}^{2} \approx 0$ holds for CE-OFDM.

\section{EMPIRICAL VALIDATION}

The VLC demonstrator used for the empirical validation of the derived formulas for the three OFDM-based waveforms under analysis consists of commercial-grade Cool-White LEDs (LUXEON Rebel LXML-PWC1-0100) in the transmitter side, and a Silicon PIN Diode detector with an embedded TIA (Thorlabs PDA100A2) in the receiver side, as shown in [15]. Two USRP N200 are used to implement the software-defined processing in the VLC transmitter and receiver, using LFTX and LFRX daughterboards $(0-30 \mathrm{MHz})$, respectively. The TIA gain can be adjusted in 10-dB steps, and the DC-bias and ACscaling factor that the LED driver applies to the OFDM-based waveform can be adjusted to minimize the clipping probability and control the LED non-linear distortion power [12], [16].
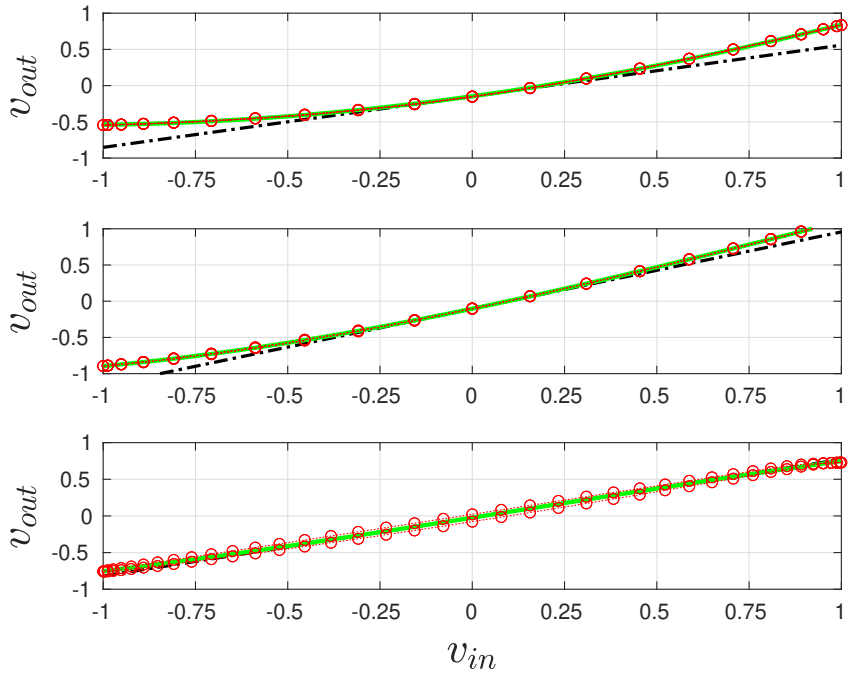

Fig. 2: Measured VLC channel transfer function for different LED DC-bias levels: $100 \mathrm{~mA}$ (top), $200 \mathrm{~mA}$ (center) and $250 \mathrm{~mA}$ (bottom). Black dashed-dotted lines show the linear response for comparison.

TABLE I: Coefficients for the third-order polynomial approximation of the LED transfer function $g_{\mathrm{LED}}(x)$ for different DC-bias currents.

\begin{tabular}{|c|c|c|c|c|}
\hline DC-bias & $c_{3}$ & $c_{2}$ & $c_{1}$ & $c_{0}$ \\
\hline $100 \mathrm{~mA}$ & -0.0154 & 0.2967 & 0.7057 & -0.1469 \\
\hline $200 \mathrm{~mA}$ & -0.0617 & 0.2067 & 1.0600 & -0.1035 \\
\hline $250 \mathrm{~mA}$ & -0.0437 & 0.0218 & 0.7955 & -0.0238 \\
\hline
\end{tabular}

The effect the DC-bias has on the LED transfer function is shown in Fig. 2, and the coefficients corresponding to the third-order polynomial approximation that minimize the mean square error are presented in Table I. As expected, for constant AC-scaling in the LED driver, using a too low (large) DCbias current increases the probability of clipping the negative (positive) peaks of the real-valued OFDM-based waveform.

\section{A. System implementation}

The software-defined VLC link was implemented in Matlab in a (few second delayed) real-time fashion, using for this purpose two computers that were connected to the two USRPs that held the LED driver (VLC transmitter) and the PD (VLC receiver). The sampling rate at both USRPs was $2 \mathrm{MS} / \mathrm{s}$ and the (I)FFT size was $N=64$. The QAM modulation order was set to $M=64$, in order to provide a trade-off solution that works fine for the three OFDM-based schemes under analysis.

Each transmission frame contained 66 OFDM symbols, as shown in Fig. 3. The first two OFDM symbols were identical synchronization words $P_{0}$, generated using a Frank-ZadoffChu (FZC) sequence with constant-amplitude envelope and optimal periodic autocorrelation properties. The FZC sequence of length $N_{\mathrm{FZC}}$ was obtained for $n=0, \ldots, N_{\mathrm{FZC}}-1$ using $\mathrm{FZC}_{n}=\exp (-\mathrm{j} \pi R n(n+2 Q+N \bmod 2) / N)$, with $R, Q \in \mathbb{N}$ and $0<R<N_{\mathrm{FZC}}$ [17]. In this paper, $N_{\mathrm{FZC}}=N / 2-1, R=11$, and $Q=0$ were used for determining the preamble sequence $P_{0}$ to feed the Hermitian Symmetry block in DCO-OFDM and CE-OFDM, whereas $N_{\mathrm{FZC}}=N-1, R=11$, and $Q=0$ were used for passband OFDM. In all cases, the DC-subcarrier was left unused. 


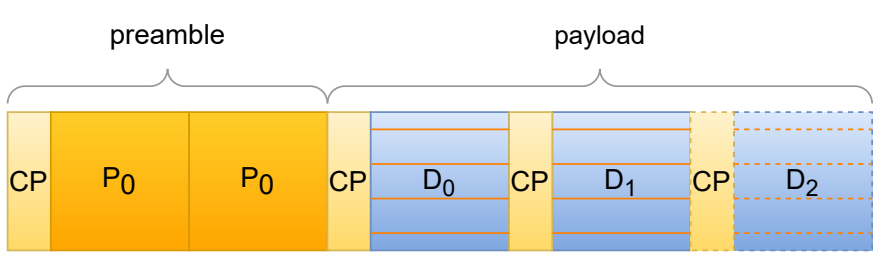

Fig. 3: OFDM frame structure consisting of 66 OFDM symbols: Two symbols for preamble $\left(P_{0}\right)$ and remaining ones for payload $\left(D_{i}\right)$. Cyclic prefix and pilot symbols (orange stripes) are also included.

TABLE II: Configuration parameters for the three OFDM schemes.

\begin{tabular}{|l|c|c|}
\hline & DCO-/CE-OFDM & Passband OFDM \\
\hline Pilots subcarriers & $\begin{array}{c}\{ \pm 7, \pm 11, \pm 15, \pm 21\} \\
\{ \pm 27, \ldots, \pm 31\} \\
\{0,-32\}\end{array}$ & $\begin{array}{c}\{ \pm 2, \pm 2, \pm 21\} \\
\{0,-32\}\end{array}$ \\
\hline Guard band & $\begin{array}{c}0 \mathrm{~Hz}(\mathrm{DCO}-\mathrm{OFDM}) \\
500 \mathrm{kHz}(\mathrm{CE}-\mathrm{OFDM})\end{array}$ & $250 \mathrm{kHz}$ \\
\hline IF carrier $\left(f_{\mathrm{IF}}\right)$ & $250 \mathrm{kHz}$ & $125 \mathrm{kHz}$ \\
\hline Baseband BW & $32 \mu \mathrm{S}$ & $64 \mu \mathrm{S}$ \\
\hline Cyclic Prefix & $128 \mu \mathrm{S}$ & $256 \mu \mathrm{S}$ \\
\hline OFDM symbol & $64-\mathrm{QAM}$ & $64-\mathrm{QAM}$ \\
\hline Carrier modulation & 22 & 50 \\
\hline Data subcarriers & $642 \mathrm{kbps}$ & $729 \mathrm{kbps}$ \\
\hline Bit rate & \multicolumn{2}{|}{} \\
\hline
\end{tabular}

The Cyclic Prefix (CP) length was set to 16 samples or, equivalently, one fourth of the FFT length. The second synchronization symbol $\left(P_{0}\right)$ did not contain $\mathrm{CP}$ to facilitate the time and frequency recovery using a Schmidl \& Cox synchronization block [18]. For DCO-OFDM and CE-OFDM (upper diagram in Fig. 1), the number of available subcarriers was reduced to $N / 2$ and, from them, $N_{\mathrm{D}}=22$ subcarriers were used for data symbols, $N_{\mathrm{P}}=4$ were reserved for QPSK pilot symbols, and the rest were use for zero-padding. Since the number of available subcarriers in passband OFDM is doubled (lower diagram in Fig. 1), $N_{\mathrm{D}}=50$ data subcarriers and $N_{\mathrm{P}}=4$ pilot subcarriers were used instead. The receive channel estimation was performed from the equal-spaced pilots using a linear least squares technique and, based on these estimates, a linear interpolation was applied to obtain the channel state information used to equalize the received signal samples on the different subcarriers.

To make fair comparisons, the data rate of the three waveforms under analysis was kept similar by adjusting the symbol time. This is because passband OFDM can accommodate about double the number of $M$-QAM symbols per OFDM frame, when compared to DCO-OFDM and CE-OFDM. For the BER measurements, the payload symbols were generated using a pseudo-random number generator with a seed that was known at both transmitter and receiver sides. Table II summarizes the parameters in the three OFDM schemes.

\section{B. Measurements and performance evaluation}

The Signal-to-Noise-plus-Distortion Ratio (SNDR) of the received signal given by

$$
\mathrm{SNDR}=\frac{\mathbb{E}\left\{|\alpha X|^{2}\right\}}{\mathbb{E}\left\{|Z|^{2}\right\}+\mathbb{E}\left\{|w|^{2}\right\}}=\frac{\alpha^{2} \sigma_{X}^{2} \mathrm{SNR}}{\sigma_{Z}^{2} \mathrm{SNR}+\sigma_{X}^{2}},
$$

where the SNR $=\mathbb{E}\left\{|X|^{2}\right\} / \mathbb{E}\left\{|w|^{2}\right\}$ is computed from the preamble using a Minimum Mean Square Error (MMSE) estimator. Thus, SNDR $\rightarrow\left(\alpha^{2} \sigma_{X}^{2}\right) / \sigma_{Z}^{2}$ as SNR $\rightarrow \infty$, where scaling factor $\alpha$ and distortion noise $\sigma_{Z}^{2}$ are functions of the coefficients $c_{i}$ used to approximate the LED nonlinear response $g_{\mathrm{LED}}(\cdot)$ when the input OFDM signal had a Root Mean Square (RMS) amplitude equal to $\sigma_{X}$. The BER curves for the different OFDM-based waveforms were obtained for three different AC-gain configurations, giving $\sigma_{X}=0.1,0.2$, and 0.3 . Once the level of non-linear distortion was set in transmission according to LED biasing point, the SNR variations to compute the BER curves in reception were obtained by changing the angle of incidence of the light beam on the PD. The theoretical lower bound for the non-distorted 64-QAM transmission is also included in the figures.

In line with the analysis presented in Section III, the effect of the LED non-linear distortion is highest for DCO-OFDM, moderate for passband-OFDM, and lowest for CE-OFDM, according to the received signal constellations shown in Fig. 4. More precisely, when studying the BER curves presented in Fig. 5a, we observe that DCO-OFDM experiences a BER floor in the $E_{\mathrm{b}} / N_{0}$ range under analysis, which grows notably even when $\sigma_{X}$ takes moderate values. For this case, the measured BER follows the theoretical lower bound rather accurately for $E_{\mathrm{b}} / N_{0}$ as high as $10 \mathrm{~dB}$ and, after that, the error floor that the theoretical analysis predicts starts to become visible.

For passband OFDM, the highest deviation between theory and practice happens at low $E_{\mathrm{b}} / N_{0}$ due to IF carrier synchronization issues (see Fig. 5b). However, this gap vanishes as the noise power is reduced, and becomes negligible for $E_{\mathrm{b}} / N_{0}$ higher than $15 \mathrm{~dB}$. As predicted in Section III-B, the effect of $g_{\mathrm{LED}}(\cdot)$ on passband OFDM is much lower than DCO-OFDM, as the higher-order distortion products falling out-of-band are eliminated by the low-pass filtering in reception. Note that, in contrast to RF, the out-of-band distortion introduced by the LED is not a serious problem as there is no adjacent-channel VLC transmission that could be affected by this impairment.

Finally, CE-OFDM was the least affected by the LED nonlinearity, and showed a very good BER performance even for input RMS signal values as high as $\sigma_{X}=1.0$. As predicted in Section III-C, since $\sigma_{Z}^{2} \approx 0$ for CE-OFDM, the received constellation plot was always the cleanest (see Fig. 4) and the $E_{\mathrm{b}} / N_{0}$ gap with respect to the lower bound BER curve was almost negligible (see Fig. 5c). Note that in the measurements that were collected, CE-OFDM enabled SNDR values on the subcarriers as high as $40 \mathrm{~dB}$, whereas in DCO-OFDM and passband OFDM the observed SNDR measurements were always below $30 \mathrm{~dB}$. Based on this, we conclude that CEOFDM can easily accommodate high-order constellations.

\section{CONCLUSION}

This paper studied the impact of the LED non-linear response on the BER performance of a VLC link based on DCOOFDM, passband OFDM, and CE-OFDM. The impact of the LED non-linear distortion was first modeled theoretically and, after that, was empirically validated using a software-defined VLC demo that relied on commercial LEDs and PIN detectors. CE-OFDM was the scheme that provided the best BER performance, followed by passband OFDM and DCO-OFDM. 


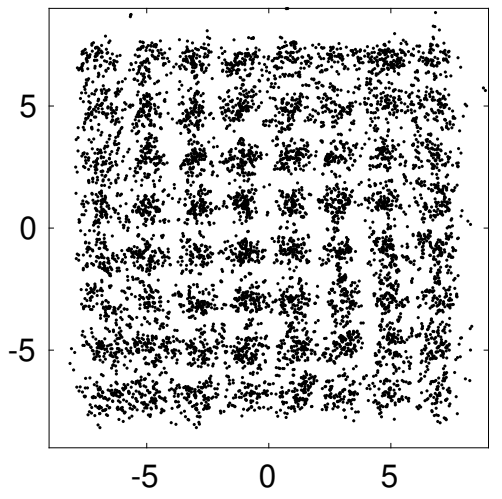

(a) DCO-OFDM waveform

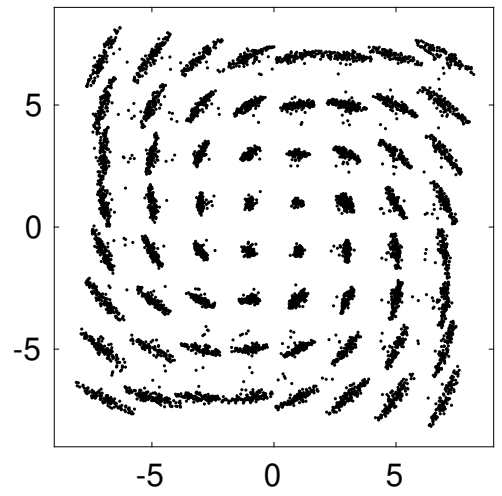

(b) Passband OFDM waveform

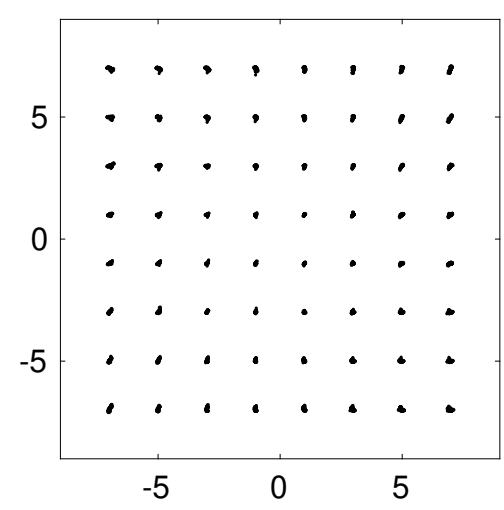

(c) CE-OFDM waveform

Fig. 4: Received constellations for the three OFDM waveforms with same DC-bias, AC-gain, and RMS amplitude of input signal $\left(\sigma_{X}=0.3\right)$.

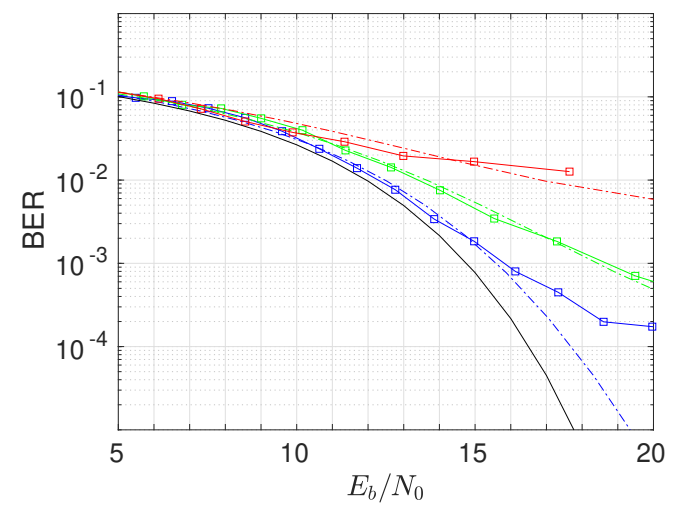

(a) DCO-OFDM waveform

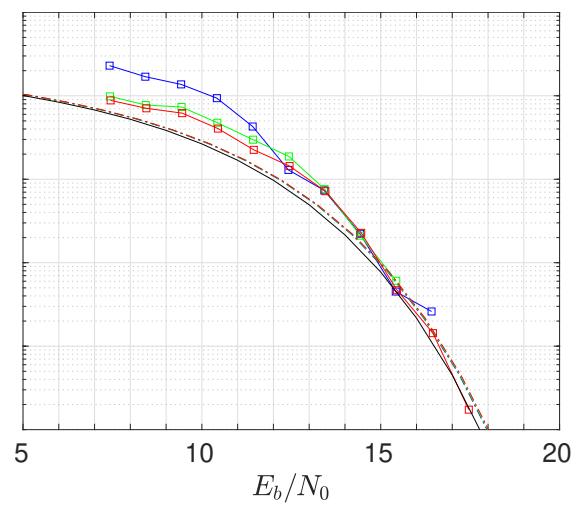

(b) Passband OFDM waveform

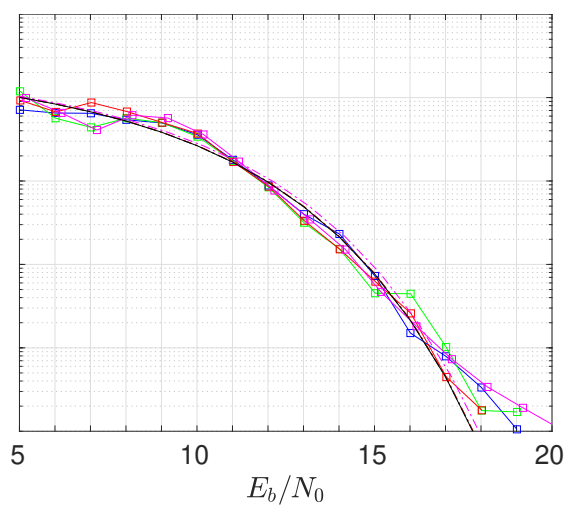

(c) CE-OFDM waveform

Fig. 5: BER for the three OFDM waveforms using 64-QAM. Square markers: Measurements. Dashed-dotted lines: Theoretical values. Solid black line: Lower bound (no distortion). Input signal RMS amplitude: $\sigma_{X}=0.1$ (blue), $\sigma_{X}=0.2$ (green), $\sigma_{X}=0.3$ (red), $\sigma_{X}=1.0$ (pink).

This is because the PAPR of CE-OFDM is constant and, due to that, the LED magnitude distortion only scales down the amplitude of the IF carrier that is phase-modulated by the real-valued OFDM waveform. Thanks to this, CE-OFDM can handle deeper intensity modulation indices, enabling to increase the fraction of the total radiant flux that is used for communications, to make the VLC system energy-efficient.

\section{REFERENCES}

[1] E. C. Strinati et al., "6G: The next frontier: From holographic messaging to artificial intelligence using subterahertz and visible light communication," IEEE Veh. Tech. Mag., vol. 14, no. 3, pp. 42-50, Sept. 2019.

[2] N. Chi, Y. Zhou, Y. Wei, and F. Hu, "Visible light communication in 6G: Advances, challenges, and prospects," IEEE Veh. Tech. Mag., vol. 15, no. 4, pp. 93-102, Dec. 2020.

[3] K. Ying et al., "Nonlinear distortion mitigation in visible light communications," IEEE Wireless Commun., vol. 22, no. 2, pp. 36-45, Apr. 2015.

[4] M. Islim and H. Haas, "Modulation techniques for Li-Fi," ZTE communications, vol. 14, no. 2, pp. 29-40, Apr. 2016.

[5] J. Armstrong, "OFDM for optical communications," J. Lightwave Tech., vol. 27, no. 3, pp. 189-204, Feb. 2009.

[6] "Standards news," IEEE Commun. Standards Mag., vol. 2, no. 4, pp. 12-17, Dec. 2018.

[7] H. Elgala, R. Mesleh, and H. Haas, "A study of LED nonlinearity effects on optical wireless transmission using OFDM," in Proc. Int. Conf. Wireless and Optical Commun. Netw., Apr. 2009, pp. 1-5.

[8] J. Armstrong and B. Schmidt, "Comparison of asymmetrically clipped optical OFDM and DC-biased optical OFDM in AWGN," IEEE Commun. Lett., vol. 12, no. 5, pp. 343-345, May 2008.
[9] H. Qian, S. Yao, S. Cai, and T. Zhou, "Adaptive postdistortion for nonlinear LEDs in visible light communications," IEEE Photonics J., vol. 6, no. 4, pp. 1-8, Aug. 2014.

[10] S. Thompson, A. Ahmed, J. Proakis, J. Zeidler, and M. Geile, "Constant envelope OFDM," IEEE Trans. Commun., vol. 56, no. 8, pp. 1300-1312, Aug. 2008.

[11] V. Singh and U. Dalal, "Integration of frequency modulated constant envelope technique with ADO-OFDM to impede PAPR in VLC," Optics Commun., vol. 418, pp. 80-87, Mar. 2018.

[12] A. Dowhuszko and A. Pérez-Neira, "Modeling the effect of non-linear distortion in a centralized RAN with analog optical fronthaul," in Proc. IEEE Global Commun. Conf., Dec. 2018, pp. 1-7.

[13] D. Dardari, V. Tralli, and A. Vaccari, "A theoretical characterization of nonlinear distortion effects in OFDM systems," IEEE Trans. Commun., vol. 48, no. 10, pp. 1755-1764, Oct. 2000.

[14] J. Bussgang, "Cross correlation functions of amplitude-distorted Gaussian signals," Tech. Rep. 216, Research Laboratory for Electronics, Massachusetts Institute of Technology, Mar. 1952.

[15] M. Ilter, A. Dowhuszko, K. Vangapattu, J. Hämäläinen, and R. Wichman, "Visible light communication-based positioning for indoor environments using supervised learning," in Proc. IEEE Global Commun. Conf., Dec. 2020, pp. 1-6.

[16] A. Dowhuszko, M. Ilter, and J. Hämäläinen, "Visible light communication system in presence of indirect lighting and illumination constraints," in Proc. IEEE Int. Conf. Commun., June 2020, pp. 1-6.

[17] R. Frank, S. Zadoff, and R. Heimiller, "Phase shift pulse codes with good periodic correlation properties," IRE Trans. Inform. Theory, vol. 8 , no. 6, pp. 381-382, Oct. 1962.

[18] T. Schmidl and D. Cox, "Low-overhead, low-complexity [burst] synchronization for OFDM," in Proc. Int. Conf. Commun., vol. 3, June 1996, pp. 1301-1306. 